\title{
EMBEDDING FREE ALGEBRAS IN SKEW FIELDS
}

\section{J. L. FISHER ${ }^{1}$}

\begin{abstract}
This paper constructs a minimal element in the partial order on the set of skew fields generated by a free algebra, and shows that the partial order contains a certain sub partial order. Examples of embedding free algebras in skew fields of heights one and two are also given.
\end{abstract}

Let $R$ be an integral domain (noncommutative) with identity. We will assume that the integral domain $R$ is always embedded in some skew field (this need not be the case in general [2], [3], [6], [7]). There are many skew fields which contain $R$ but in the commutative case there is only one field which is generated by $R$, namely the field of fractions. In the noncommutative case there may be several distinct skew fields generated by $R$ [4, pp. 277]. For skew fields $D_{1}$ and $D_{2}$ generated by $R$, we say $D_{1} \geqq D_{2}$ if there exists a place from $D_{1}$ to $D_{2}$ which extends the natural isomorphism between the embeddings of $R$. This paper shows that this is a partial order on the set $P$ (we identify isomorphic embeddings) of skew fields generated by $R$ and in the case where $R$ is the free algebra on two generators we show that $P$ contains the subposet $\mathscr{L}$ where $C(x)$ is the unique maximal element $[1$, Theorem 27] of $\rho$ and $K_{i}$ are distinct elements of $\rho$ with $K_{2}$ minimal in $\rho$. We also examine the height of an integral domain and give examples of embeddings of different heights of a free algebra in two skew fields.

I. The partial order and the chain of domains $Q_{i}(K, \phi(R))$.

Definition. Let $D$ be a skew field and $\phi$ an isomorphism of $R$ into $D$. $R$ is fully embedded in $D$ if the smallest sub skew field of $D$ containing $\phi(R)$ is $D$ itself. We will denote a full embedding of $(D, \phi(R))$ by $(D, \phi)$.

Definition. If $D_{1}$ and $D_{2}$ are two division rings we say $\phi$ is a place from $D_{1}$ to $D_{2}$ if $\phi$ is a homomorphism from a local subring $S$ of $D_{1}$ onto $D_{2}$ [local means the set of nonunits is an ideal ].

Definition. For full embeddings $(D, \alpha)$ and $(K, \gamma)$ we say $(D, \alpha)$ $\geqq(K, \gamma)$ if there is a place $\phi$ from $D$ to $K$ such that $\phi^{-1}(K) \supset \alpha(R)$ and $\phi \mid \alpha(R)=\gamma \alpha^{-1}$.

Received by the editors October 9, 1970.

AMS 1969 subject classifications. Primary 1646, 1615.

Key words and phrases. Noncommutative integral domain, height of a domain embedding free algebras, skew fields.

1 Research supported by the National Research Council of Canada.

Copyright ( 1971, American Mathematical Society 
$(D, \alpha) \cong(K, \gamma)$ if there is an isomorphism $\phi$ between $D$ and $K$ such that $\phi \mid \alpha(R)=\gamma \alpha^{-1}$. If we identify isomorphic embeddings the class of embeddings is a set.

We also make the following notation. Suppose $\phi$ is an isomorphism of $R$ into a skew field $K$.

$Q_{0}(K, \phi(R))=\phi(R)$.

$Q_{1}(K, \phi(R))=$ subring of $K$ generated by $\left\{a, b^{-1}\right.$ such that $a, b$ $\in Q_{0}(K, \phi(R))$ with $\left.b \neq 0\right\}$.

Inductively we define

$Q_{i}(K, \phi(R))=$ subring of $K$ generated by $\left\{a, b^{-1}\right.$ such that $a, b$ $\in Q_{i-1}(K, \phi(R))$ with $\left.b \neq 0\right\}$.

When no confusion arises, we will write $Q_{i}, Q_{i}(K, \phi)$ or $Q_{i}(\phi(R))$ for $Q_{i}(K, \phi(R))$.

It is easy to see that $\bigcup_{i} Q_{i}(K, \phi(R))$ is the skew subfield of $K$ generated by $R$ and $\left(\cup Q_{i}, \phi\right)$ is a full embedding.

LEMмA 1 . The relation $\geqq$ is a partial order on the set of full embeddings $\rho$ of $R$.

Proof. We will show that if $\left(D_{1}, \alpha\right) \geqq\left(D_{2}, \gamma\right)$ and $\left(D_{2}, \gamma\right) \geqq\left(D_{1}, \alpha\right)$ that $\left(D_{1}, \alpha\right) \cong\left(D_{2}, \gamma\right)$. The rest is clear. Let $\phi_{1}$ be a place from $S_{1}$ onto $D_{2}, D_{1} \supset S_{1} \supset \alpha(R)$ and $\phi_{2}$ a place from $S_{2}$ onto $D_{1}, D_{2} \supset S_{2} \supset \gamma(R)$. $\phi_{1}\left|\alpha(R)=\gamma \alpha^{-1}=\left(\alpha \gamma^{-1}\right)^{-1}=\phi_{2}^{-1}\right| \alpha(R)$. Hence $r \neq 0 \in Q_{0}\left(D_{1}, \alpha\right)$ implies $\phi_{1}(r) \neq 0 \Rightarrow r^{-1} \in S_{1}$ so that $S_{1} \supset Q_{1}\left(D_{1}, \alpha\right) . \phi_{1} \mid Q_{1}\left(D_{1}, \alpha\right)$ is an isomorphism since $\phi_{1}\left(\sum m_{i}\right)=0$, where $m_{i}$ is a product of elements of $\alpha(R)$ and inverses of elements of $\alpha(R)$, implies $\sum m_{i}=\phi_{2}\left(\sum \phi_{2}^{-1}\left(m_{i}\right)\right)$ $=\phi_{2}\left(\sum \phi_{1}\left(m_{i}\right)\right)=\phi_{2}\left[\phi_{1}\left(\sum m_{i}\right)\right]=0 . \phi_{1}=\phi_{2}^{-1}$ on $Q_{1}\left(D_{1}, \alpha\right)$. Similarly $\phi_{1}$ is an isomorphism on all $Q_{i}$ and hence

$$
D_{1}=U Q_{i}\left(D_{1}, \alpha\right) \stackrel{\phi_{1}}{\cong} \cup Q_{i}\left(D_{2}, \gamma\right)=D_{2} .
$$

LEMмA 2. If $(K, \alpha)$ is a full embedding and $Q_{i}(K, \alpha)$ is simple for each $i \geqq 1$ then $(K, \alpha)$ is minimal in $\beta$.

Proof. Suppose $(K, \alpha) \geqq(D, \gamma)$. Hence $\phi$ is a homomorphism from a local subring $S$ of $K$ onto $D . \phi \mid \alpha(R)=Q_{0}$ is an isomorphism. Suppose $\phi \mid Q_{j}$ is an isomorphism. Hence $Q_{j+1} \subset S$ and $\phi$ maps $Q_{j+1}(K, \alpha)$ onto $Q_{j+1}(D, \gamma)$. Since $Q_{j+1}(K, \alpha)$ is simple $\phi$ is an isomorphism on $Q_{j+1}$. Hence $\phi$ is an isomorphism from $\cup Q_{i}=K$ onto $D$.

Our last general observation is the following. Let $I$ be an ideal of $Q_{i+1}$, and let $i$ be the inclusion of $Q_{i}$ into $Q_{i+1}$. This induces a monomorphism $i$ from $Q_{i}$ into $Q_{i+1} / I$. This is clear since every element of $Q_{i}$ has an inverse in $Q_{i+1}$ and hence in $Q_{i+1} / I . Q_{i}$ and its inverses still generate $Q_{i+1} / I$. 
II. The $K$-height of a free algebra in two different skew fields. Suppose $\phi$ is an isomorphism of $R$ in to a skew field $K$. The $K$-height of $\phi(R)$ is $h_{K}(\phi(R))=\min \left\{i: Q_{i}(K, \phi(R))\right.$ is a skew field $\}$ or $\infty$ if $Q_{i}$ is not a skew field for any $i$.

Denote the free algebra on a set $X$ over the field $F$ by $F[X]$. B. H. Neumann [8] gave an example of an embedding of $F[X]$ in a skew field $D$ and conjectured that $h_{D}(F[X])=\infty$. Jategaonkar [5] gives a different embedding. We show that this has height 2 and construct another embedding of height one.

Let $Q$ be the rationals and let $F=Q\left(t_{1}, t_{2}, \cdots\right)$ be the commutative field with indeterminates $t_{1}, t_{2}, \cdots$. Denote the skew polynomial ring $F[x ; \sigma]$ by $S$ where $\sigma$ is the monomorphism of $F$ into $F$ induced by mapping $t_{i}$ into $t_{i+1}$, and where $\sigma\left(t_{i}\right) x=x t_{i}$. S is embedded in the division ring $K=\left\{x^{-n} \sum_{i=0}^{\infty} f_{i} x^{i}\right.$ where $\left.f_{i} \in F\right\}$. Since $x S+t_{1} x S$ is direct, the ring $R=Q\left[x, t_{1} x\right]$ is a free algebra over $Q$ and embedded in $K$ (Jategaonkar [5]).

THEOREM 1. $h_{K}(R)=2$.

We will use the following series of lemmas to prove this.

LemMa 3. $Q_{2}(R)$ is a skew field.

Proof. The subring $Q_{1}(R)$ contains $x$ and $x^{-1}$ and hence contains $t_{i}=x^{i-1}\left(t_{1} x\right) x^{-i}$. Thus $Q_{1}(R) \supset Q\left[t_{1}, \cdots\right][x ; \sigma]$ which is left Ore with a ring of quotients $K_{1}$. Thus taking quotients again we get $Q_{2}(R)$ $\supset K_{1} \supset Q_{1}(R)$. Since $K_{1}$ is a field, by taking quotients again we have $Q_{3}(R) \supset K_{1} \supset Q_{2}(R)$ and the lemma is proven.

LEMmA 4. If $r \in R$ then $r=\sum f_{i} x^{i}$ where $f_{i}=\sum_{I} \alpha_{I} t_{1}^{\epsilon_{1}} t_{2}^{\epsilon_{2}} \cdots t_{i}^{\epsilon_{i}}$, $\alpha_{I} \in Q$, where I ranges over all $i$-tuples $\left(\epsilon_{1}, \cdots, \epsilon_{i}\right)$ with $\epsilon_{j}=1$ or 0 .

Proof. Since $R$ is a free algebra it is sufficient to examine a monomial $m$. We claim that if $m$ is of degree $i$ then $m=t_{1}^{\epsilon_{1}} t_{2}^{\epsilon_{2}} \cdots t_{1}^{\epsilon_{i}} x^{i}$ where $\epsilon_{j}=1$ or 0 . If $i=1$ then this is true, since the only monomials we have are $\alpha x$ and $\beta t_{1} x$ where $\alpha, \beta \in Q$. Suppose true for $i \leqq n$. If $m$ is of degree $n+1$, then $m=m^{\prime} t_{1}^{\epsilon_{n+1}} x$ where $m^{\prime}$ has degree $n$, and $\epsilon_{n+1}$ is either 1 or 0 . By induction $m^{\prime}=t_{1}^{\epsilon_{1}} t_{2}^{\epsilon_{2}} \cdots t_{n}^{\epsilon_{n}} x^{n}$ and $m=t_{1}^{\epsilon_{1}} t_{2}^{\epsilon_{2}} \cdots t_{n}^{\epsilon_{n}} t_{n+1}^{\epsilon_{n+1}} x^{n+1}$. Gathering monomials of same degree we have the lemma.

The following notation will be used in the remaining lemmas. Define $\delta=\left\{\sum_{I} \alpha_{I} t_{1}^{\epsilon_{1}} t_{2}^{\epsilon_{2}} \cdots t_{i}^{\epsilon_{i}} \neq 0\right.$ where $I$ ranges over all $i$-tuples $\left(\epsilon_{1}, \cdots, \epsilon_{i}\right)$ where $\epsilon_{j}=1$ or 0 , and $i$ ranges over all integers $\}$. Let $\mathbb{T}$ be all products of elements of $\delta$. It is easy to see that $\sigma(\delta) \subset \delta$ and thus $\sigma(\mathfrak{T}) \subset \mathfrak{T}$. Since $\mathscr{N}$ is multiplicatively closed, let $V=Q\left[t_{1}, t_{2}, \cdots\right]$ localized about $\mathfrak{T}$. $\sigma(V) \subset V$ and from this it is not difficult to see that $U=\left\{x^{-n} \sum_{i=0}^{\infty} v_{i} x^{i} \mid v_{i} \in V\right\}$ is a subring of $K$. 
Lemma 5. $Q_{1}(R) \subset U$.

Proof. It is sufficient to show that $r \in R$ implies $r^{-1} \in U$. Let $r=\sum_{i=0}^{n} f_{i} x^{i+i_{0}}$ where $f_{0} \neq 0 \quad$ and $f_{i} \in \mathcal{S} . \quad r^{-1}=x^{-i_{0}}\left(\sum_{i=0}^{n} f_{i} x^{i}\right)^{-1}$ $=x^{-i_{0}} \sum_{i=0}^{\infty} b_{i} x^{i}$ where $f_{0} b_{0}=1$, and $b_{n}=-f_{0}^{-1} \sum_{i=1}^{n} f_{i} \sigma^{i}\left(b_{n-i}\right)$. Since $b_{0}=f_{0}^{-1} \in V$ and $\sigma(V) \subset V$ we have by induction that $b_{n} \in V$. Thus $r^{-1} \in U$.

L.EMMA 6. $\left(t_{1}-t_{2}^{2}\right)^{-1}$ is not contained in $U$.

Proof. If $\left(t_{1}-t_{2}^{2}\right)^{-1}$ were in $U$ then it would be in $V$. Thus $\left(t_{1}-t_{2}^{2}\right)^{-1}=f / g$ where $g \in \mathscr{N}$ and $f \in Q\left[t_{1}, \cdots\right]$. Therefore $g=f\left(t_{1}-t_{2}^{2}\right)$ where $t_{1}-t_{2}^{2}$ is irreducible and hence divides $g=\pi p_{i}$ with $p_{i} \in \mathcal{S}$. This is impossible however since no $p_{i}$ has terms with square factors.

The theorem now follows since $\left(t_{1}-t_{2}^{2}\right)^{-1} \notin Q_{1}(R)$ implies $Q_{1}(R)$ is not a skew field.

We continue with the same notation, and construct an embedding of height one by factoring out a maximal ideal of $Q_{1}(K, R)$. Let $P$ be the ideal of $Q\left[t_{1}, t_{2}, \cdots\right]$ generated by $t_{1}-t_{2}^{2}, t_{2}-t_{3}^{2}, \cdots$. It is clear that $\sigma(P) \subset P$. It is also not difficult to see that

$$
Q\left[t_{1}, \cdots\right] / P \cong Q\left[\bar{t}_{1}, \bar{t}_{1}^{1 / 2}, \bar{t}_{1}^{1 / 4}, \cdots\right]
$$

and hence $P$ is a prime ideal. $\sigma$ extends to an automorphism of $Q\left[\bar{t}_{1}, \bar{t}_{1}^{1 / 2}, \cdots\right]$ where $\sigma\left(\bar{t}_{1}^{1 / 2^{n}}\right)=\bar{t}_{1}^{1 / 2^{n+1}}$. In $Q\left[t_{1}, \cdots\right] / P$ every element can be written uniquely as $\sum_{k} \sum_{I} \alpha_{I, k} t^{\bar{t}_{2}^{\epsilon_{1}}} \ldots \bar{t}_{n+1}^{\epsilon_{n}}$ so that $\mathfrak{M} \cap P$ $=\varnothing$. Therefore factoring out the ideal of $Q_{1}\left(Q\left[x, t_{1} x\right]\right)$ generated by $P$ we get the free algebra $Q\left[x, t_{1} x\right]$ embedded in the Ore domain $Q\left[\bar{t}_{1}, \bar{t}_{1}^{1 / 2}, \bar{t}_{1}^{1 / 4}, \ldots\right][x ; \sigma]$ and thus in a skew field, namely the domain's field of quotients $K_{2}$.

We will write $t$ for $\bar{t}_{1}$.

THEOREM 2. $Q_{1}\left(K_{2}, Q[x, t x]\right)$ is a division ring.

Proof. Since $x$ and $x^{-1}$ are in $Q_{1}$, all integral powers of $x$ are in $Q_{1}$. We first observe that if $p(x) \in Q\left[t, t^{1 / 2}, \cdots\right][x ; \sigma]$ then there exists integers $n$ and $m$ such that $x^{n} p(x) x^{m} \in Q[x, t x]$. Suppose $p(x)=\sum_{i=0}^{k} a_{i} x^{i}$ where

$$
a_{i}=\sum_{j=0}^{k_{i}}\left(\sum_{I} \alpha_{I, i, j} t^{\epsilon_{1} / 2} \cdots t^{\epsilon_{m} / 2^{m}}\right) t^{j}
$$

where $I$ runs over the $m$-tuples $\left(\epsilon_{1}, \cdots, \epsilon_{m}\right)$ with each $\epsilon_{l}=0$ or 1 . Pick $n$ to be an integer such that $2^{n} \geqq \max \left\{k_{i}\right\}$.

Therefore $x^{n} p(x)=\sum_{i=0}^{k} a_{i}^{\prime} x^{i+n}$ where each 


$$
a_{i}^{\prime}=\sum_{j=0}^{k_{i}}\left(\sum_{I} \alpha_{I, i, j} t^{\epsilon_{1} / 2 n+1} \cdots t^{\epsilon_{m} / 2 n+m}\right) t^{j / 2 n} .
$$

Each $j$ can be written in the form $\gamma_{n}+\gamma_{n-1} 2+\cdots+\gamma_{0} 2^{n}$ with $\gamma_{i}=0$, or 1 . Hence

$$
t^{j / 2 n} t^{\epsilon_{1} / 2^{n+1}} \cdots t^{e_{m} / 2^{n+m}}=t^{\gamma_{0} t^{\gamma_{1} / 2}} \cdots t^{\gamma_{n} / 2{ }^{n}} t^{\epsilon_{1} / 2 n+1} \cdots t^{e_{m} / t^{n+m}}
$$

where $\left(\gamma_{0}, \gamma_{1}, \cdots, \gamma_{n}, \epsilon_{1}, \cdots, \epsilon_{m}\right)$ is an $n+m$ tuple of zeros and ones. Hence $x^{n} p(x) x^{m} \in Q[x, t x]$.

Since $R=Q\left[t, t^{1 / 2}, \cdots\right][x ; \sigma]$ is Ore and contained in $Q_{1}$ we may assume that an element of $Q_{1}$ is written $q(x)^{-1} p(x)$ with $q(x)$ and $p(x) \in R$.

By the above we can choose integers $n$ and $m$ such that both $x^{n} p(x) x^{m}$ and $x^{n} q(x) x^{m}$ are in $Q[x, t x]$.

Hence $\left(x^{n} p(x) x^{m}\right)^{-1} \in Q_{1}$ and

$$
1=\left(q(x)^{-1} p(x)\right)\left[x^{m}\left(x^{n} p(x) x^{m}\right)^{-1}\left(x^{n} q(x) x^{m}\right) x^{-m}\right] \in Q_{1}
$$

so that $Q_{1}$ is a division ring.

COROLlaRY $1 . h_{K_{2}}(Q[x, t x])=1$.

III. The poset of skew fields. Let $K^{\prime}$ be the field of quotients of $Q\left(t_{1}, t_{2}, \cdots\right)[x ; \sigma]$ where $\sigma\left(t_{i}\right)=t_{i+1}$ and $x f=\sigma(f) x$ for $f \in Q\left(t_{1}, t_{2}, \cdots\right)$. Let $F_{n}$ be $Q\left[t_{1}, t_{2}, \cdots\right]$ localized about the prime ideal $P_{n}=\left\langle t_{1}-t_{2}^{n}, t_{2}-t_{3}^{n}, \cdots\right\rangle$ for a positive integer $\left.n\right\rangle$. $\sigma\left(P_{n}\right) \subset P_{n}$ so we can localize $F_{n}[x ; \sigma]$ about the ideal generated by $P_{n}$, to obtain $S_{n}$. $S_{n}$ is a local ring which maps onto the field of quotients $K_{n}$ of $Q\left(s, s^{1 / n}, s^{1 / n^{2}}, \cdots\right)[y ; \sigma]$. If $Q[u, v]$ is the free algebra on two generators, the map $\phi(u)=x$ and $\phi(v)=t_{1} x$ maps $Q[u, v]$ onto a free algebra generating $K^{\prime}$. Likewise $\phi_{n}(u)=y$ and $\phi_{n}(v)=s y$ maps $Q[u, v]$ onto a free algebra generating $K_{n}$.

THEOREM 3. $\left(K_{n}, \phi_{n}\right) \geq\left(K_{m}, \phi_{m}\right)$ for $n \neq m$.

Proof. The quotient field of $Q\left(s, s^{1 / n}, s^{1 / n^{2}}, \cdots\right)[y ; \sigma]$ is $K_{n}$ where $y s=s^{1 / n} y$ and the quotient field of $Q\left(r, r^{1 / m}, r^{1 / m^{2}}, \cdots\right)[z ; \sigma]$ is $K_{m}$ where $z r=r^{1 / m} z$. If $\left(K_{n}, \phi_{n}\right) \geqq\left(K_{m}, \phi_{m}\right)$ and $\gamma$ is the place then $\gamma(s)$ $=\gamma\left(s y y^{-1}\right)=r z z^{-1}=r$ but $\gamma\left(s^{1 / n}\right)=\gamma\left(y s y y^{-2}\right)=z r z z^{-2}=r^{1 / m} \cdot \gamma\left[\left(s^{1 / n}\right)^{n}\right]$ $=\left(r^{1 / m}\right)^{n}=r$ which implies $n=m$.

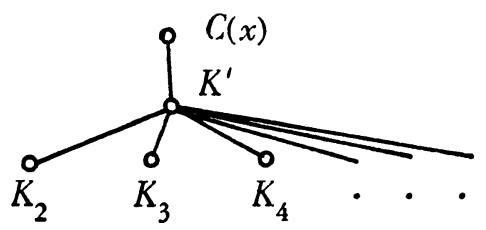


Hence we have $\left(K^{\prime}, \phi\right)>\left(K_{n}, \phi_{n}\right)$ for each $n>1$. Furthermore, since $Q_{1}\left(K_{2}, \phi_{2}\right)$ is a skew field and hence simple, Lemma 2 says $\left(K_{2}, \phi_{2}\right)$ is minimal. By [1, Theorem 27], $\odot$ has a maximal element $C(x)$ and we have the subposet $\mathscr{L}$ embedded in $P$.

\section{REFERENCES}

1. S. A. Amitsur, Rational identities and applications to algebra and geometry, J. Algebra 3 (1966), 304-359. MR 33 \#139.

2. L. A. Bokut', Embedding rings into skew fields, Dokl. Akad. Nauk SSSR 175 (1967), 755-758 =Soviet Math. Dokl. 8 (1967), 901-904. MR 36 \#5167.

3. A. J. Bowtell, On a question of $\mathrm{Mal}^{\prime} \mathrm{cev}$, J. Algebra 7 (1967), 126-139. MR 37 $\# 6310$.

4. P. M. Cohn, Universal algebra, Harper \& Row, New York, 1965. MR 31 \#224.

5. A. V. Jategaonkar, Ore domains and free algebras, Bull. London Math. Soc. 1 (1969), 45-46. MR 39 \#241.

6. A. A. Klein, Rings nonembeddable in fields with multiplicative semigroups embeddable in groups, J. Algebra 7 (1967), 100-125. MR 37 \#6309.

7. A. I. Mal'cev, On the immersion of an algebraic ring into a field, Math. Ann. 113 (1937), 686-691.

8. B. H. Neumann, On ordered division rings, Trans. Amer. Math. Soc. 66 (1949), 202-252. MR 11, 311.

University of Alberta, Edmonton, Alberta, Canada 